\title{
Effects of inlet boundary conditions, on the computed flow in the Turbine-99 draft tube, using OpenFOAM and CFX
}

\author{
$\mathrm{H}_{\text {Nilsson }}{ }^{1}$ and $\mathrm{M} \mathrm{J}$ Cervantes $^{2}$ \\ ${ }^{1}$ Chalmers University of Technology, SE-412 96 Gothenburg, Sweden \\ ${ }^{2}$ Luleå University of Technology, SE-971 87 Luleå, Sweden \\ E-mail: michel.cervantes@1tu.se
}

\begin{abstract}
The flow in the Turbine-99 Kaplan draft tube was thoroughly investigated at three workshops $(1999,2001,2005)$, which aimed at determining the state of the art of draft tube simulations. The flow is challenging due to the different flow phenomena appearing simultaneously such as unsteadiness, separation, swirl, turbulence, and a strong adverse pressure gradient. The geometry and the experimentally determined inlet boundary conditions were provided to the Turbine-99 workshop participants. At the final workshop, angular resolved inlet velocity boundary conditions were provided. The rotating non-axi-symmetry of the inlet flow due to the runner blades was thus included. The effect of the rotating angular resolution was however not fully investigated at that workshop. The first purpose of this work is to further investigate this effect. Several different inlet boundary conditions are applied - the angular resolved experimental data distributed at the Turbine-99 workshop, the angular resolved results of a runner simulation with interpolated values using different resolution in the tangential and radial directions, and an axi-symmetric variant of the same numerical data. The second purpose of this work is to compare the results from the OpenFOAM and CFX CFD codes, using as similar settings as possible. The present results suggest that the experimental angular inlet boundary conditions proposed to the workshop are not adequate to simulate accurately the flow in the T-99 draft tube. The reason for this is that the experimental phaseaveraged data has some important differences compared to the previously measured timeaveraged data. Using the interpolated data from the runner simulation as inlet boundary condition however gives good results as long as the resolution of that data is sufficient. It is shown that the difference between the results using the angular-resolved and the corresponding symmetric inlet data is very small, suggesting that the importance of the angular resolution is small. The results from OpenFOAM and CFX are very similar as long as the inlet data resolution is fine enough. CFX seems to be more sensitive to that resolution.
\end{abstract}

\section{Introduction}

Since 1999, there have been three workshops on the flow simulation in the Hölleforsen Kaplan draft tube model, here referred to as the Turbine-99 draft tube. At the third workshop in 2005 [1], the participants were offered the possibility to apply angular resolved boundary conditions at the draft tube inlet from phase-averaged LDA measurements. One such contribution was made [2], in which the experimental data close to the hub was modified according to the behaviour of earlier time-averaged data. Those results turned out be quite close to the experimental data further downstream in the draft tube, and it was argued that the resolution of the runner blade wakes was the reason for the success. A work was presented some years later by Cervantes et al. [3] using the same inlet boundary conditions 
with imposed rotation to simulate the upstream presence of the runner. Mesh sensitivity analysis was performed. A discrepancy was found below the runner cone between the simulated and experimental results further downstream, while the unsteady wall pressure compare well. The velocity discrepancy needed further attention through a more detailed investigation of the inlet boundary condition.

The present work investigates the effect of the inlet boundary condition on the flow in the Turbine99 draft tube. Both the originally proposed phase-averaged experimental data, and data obtained from separate runner simulations are used as inlet boundary condition. The numerical data includes the effect of hub and tip clearance flow, and includes all the required data all the way to the walls, contrary to the experimental data which is lacking some information; radial velocity and corresponding fluctuation. The difference between the results from angular resolved and axisymmetric data derived from the same numerical dataset is discussed. The simulations are done in two industrially used CFD codes, OpenFOAM-1.5-dev and ANSYS CFX 13, using as similar settings as possible. The differences between the results from those codes are discussed.

\section{Mesh, turbulence model and boundary conditions}

The present simulations have been done on a block-structured hexahedral mesh with 1216488 cells, of the same topology that was originally distributed at the Turbine-99 workshops. The mesh was constructed in ICEM CFD, and the characteristics of the grids include a minimum face angle of $18.8^{\circ}$, maximum expansion factor of 55 and maximum aspect ratio of 16288. The boundary layers are resolved in the present work, for the use with the Low-Re k-Omega SST turbulence model. Typical maximum $\mathrm{y}^{+}$values are 0.05 and 0.55 in OpenFOAM and CFX, respectively. As the angular resolved inlet boundary conditions are rotating, simulating the presence of a runner upstream, special attention was given to the inlet to avoid fluctuations due to interpolation discrepancies. The distance between 2 consecutive nodes in the angular direction at each radius was constant at the inlet, to reduce the effects of interpolation at each time step.

Two different sources for the draft tube inlet boundary condition at cross-section Ia (see figure 1) have been used, the phase-averaged LDA measurements by Andersson [4], and interpolated results of the runner simulation done using OpenFOAM by Nilsson [5]. The boundary conditions, normalized with the experimental bulk velocity (based on $\mathrm{Q}=0.522 \mathrm{~m}^{3} / \mathrm{s}$ ), are shown in figures 2 and 3 , and are here discussed.

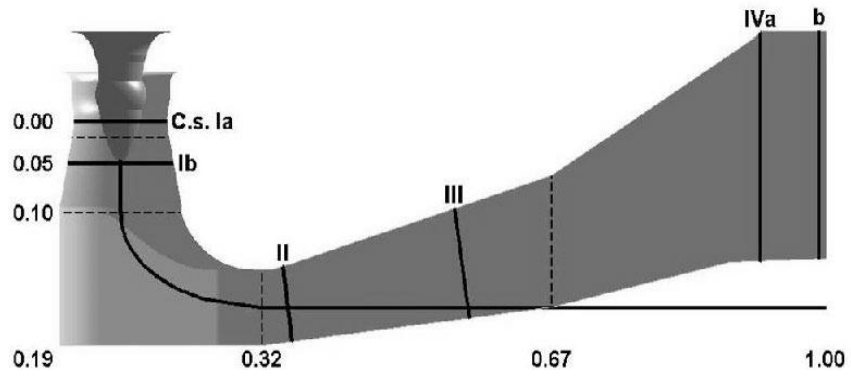

Figure 1. Draft tube model used for the Turbine-99 workshops. The computational domain inlet boundary is located at section Ia, where rotating angular resolved and symmetric boundary conditions are applied. The results are evaluated at sections $\mathrm{Ib}$ and Ic (below Ib).

The experimental inlet boundary condition is derived from the phase-averaged LDA measurements performed by Andersson [4] at cross-section Ia, see Fig. 1. The measurements were done along a single line, perpendicular to the shroud wall, and the angular resolved data was reconstructed using a runner trigger signal. The measurements provide phase-averaged axial (U, normal to the measurement line) and tangential (V) velocity components, as well as the RMS in the axial ( $\left.u^{\prime}\right)$ and tangential ( $\left.v^{\prime}\right)$ directions. Some details are missing in order to use the experimental data as inlet boundary condition, and the assumptions made in the present work are:

- The experimental data was taken along a line perpendicular to the shroud, but directly applied to the flat draft tube inlet, and the measured axial component was assumed to be the true axial component, as suggested at the Turbine-99 workshops. At the Turbine-99 workshops it was suggested to multiply the velocity vectors by 1.07 to correct the volume flow, which was also 
done in the present work. This yields a volume flow of $0.506 \mathrm{~m}^{3} / \mathrm{s}$, i.e. less than the measured value of $0.522 \mathrm{~m}^{3} / \mathrm{s}$.

- The experimental data has been extrapolated to the walls at cross-section Ia, using a linear variation to the appropriate wall value for each variable. The radius of the hub and shroud are $98.09 \mathrm{~mm}$ and $236.46 \mathrm{~mm}$, respectively, and the measured point closest to the hub and shroud are at radius $109.49 \mathrm{~mm}$ and $234.05 \mathrm{~mm}$, respectively. It should be noted that the extrapolation to the hub is over a rather long distance, and the measured data closest to the hub also lacks a peak in the axial velocity, which was present in the time-resolved data (and in the numerical data). The flow in that region is of great importance for the flow in the draft tube.

- The radial velocity component is computed according to what was originally suggested by Bergström [5] and proposed at the later workshops [1], i.e:

$$
V=U \cdot \tan (\alpha) \text {, }
$$

where

$$
\alpha=\alpha_{\text {cone }}+\left(\frac{\alpha_{\text {wall }}-\alpha_{\text {cone }}}{R_{\text {wall }}-R_{\text {cone }}}\right) \cdot\left(r-R_{\text {wall }}\right)
$$

$\alpha_{\text {wall }}=-12.8^{\circ}, \alpha_{\text {wall }}=2.8^{\circ}, R_{\text {wall }}=236.46 \mathrm{~mm}$ and $R_{\text {cone }}=98.09 \mathrm{~mm}$.

- The turbulent kinetic energy is calculated from the measured RMS data as $\mathrm{k}=1 / 2\left(\mathrm{u}^{\prime} \wedge 2+2 * \mathrm{v}^{\prime \wedge} 2\right)$. It is thus here assumed that $\mathrm{w}^{\prime}=\mathrm{v}^{\prime}$. The RMS values have not been modified according to the velocity correction of 1.07.

- The turbulence eddy frequency is computed as $\omega=\mathrm{k}^{\wedge} 0.5 /\left(\mathrm{C}_{\mathrm{mu}} \cdot 1\right)$, where the eddy length scale is assumed as $1=0.1 \mathrm{~m}$, and $\mathrm{C}_{\mathrm{mu}}=0.09$, as recommended in CFX.

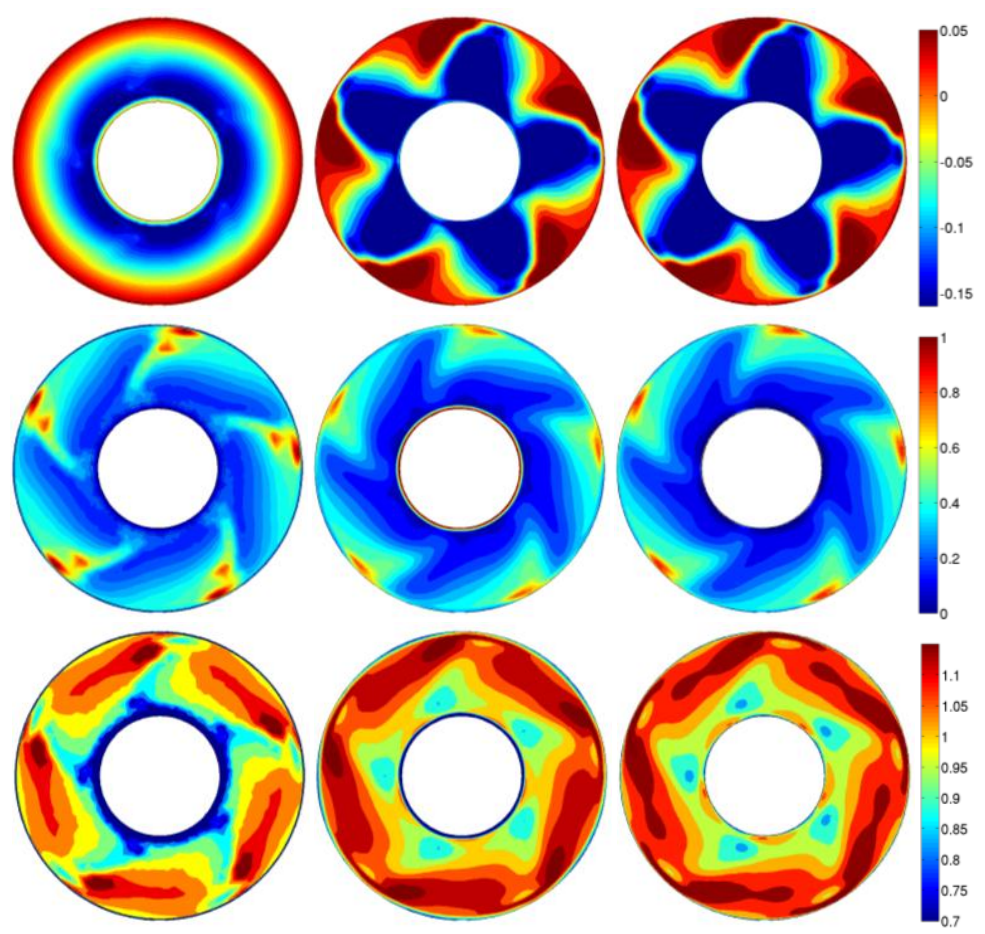

Figure 2. Measured and computed angular resolved normalized velocity distribution at section Ia, extrapolated to hub and shroud. Left: Measured, Center: Coarse computed, Right: Fine computed. Row 1: Radial. Row 2:

Tangential. Row 3: Axial. 

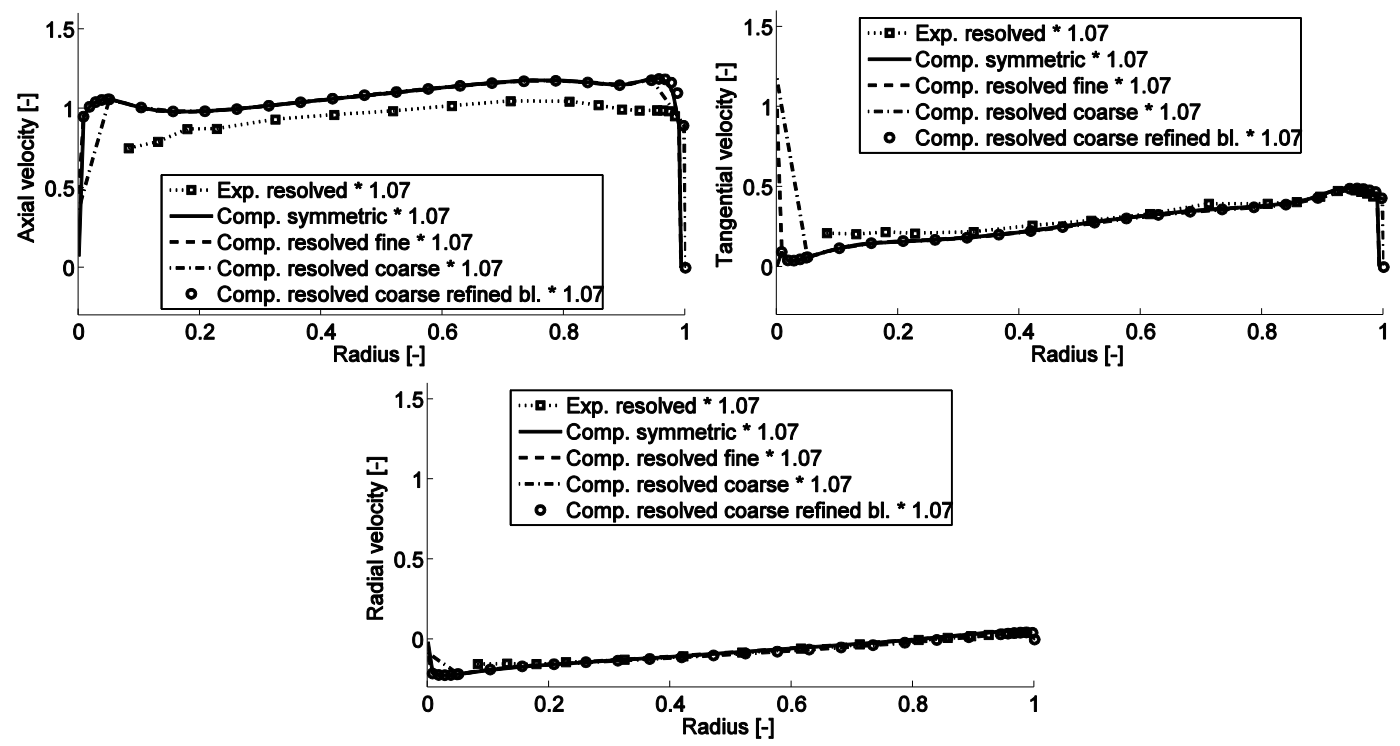

Figure 3. Circumferentially averaged representation of the inlet boundary conditions.

The draft tube inlet boundary condition derived from the separate runner results were taken at the exact location of cross-section Ia, and applying the same velocity correction of 1.07 as in the experiments, yielding a volume flow of $0.556 \mathrm{~m}^{3} / \mathrm{s}$ which is more than the measured value of 0.522 $\mathrm{m}^{3} / \mathrm{s}$. The runner results were steady in the rotating frame of reference, and axi-periodic, making it possible to create the same kind of data as from the experiments using sampling on a cylindrical fine mesh of 100 radial and 360 tangential points, and a coarse mesh of 22 radial and 120 tangential points. A third mesh was constructed from the coarse mesh with some additional radial positions to capture some specific flow features, as shown in figure 3. The fine mesh and the coarse mesh with resolved boundary layer are very similar, both in terms of circumferentially averaged values and in the angular resolved distribution. The circumferentially averaged values of the fine mesh were used to create the axi-symmeric boundary condition that was used in one of the present simulations. A small extrapolation from the radial end sampling points, to the appropriate wall values was done to avoid possible problems with uncovered inlet faces in the resolved boundary layer. From the sampling, the radial, tangential and axial velocity components, and the turbulent kinetic energy and turbulence eddy dissipation were given. The turbulence eddy frequency was then calculated in the interpolated points as $\omega=\mathrm{k}^{0.5} / \mathrm{C}_{\mathrm{mu}} / 1$, as recommended in CFX. It should be noted that the runner mesh was designed for wall-functions, i.e. the sampled values in the region very close to the wall are only approximate.

When using the angular resolved boundary conditions the values between the measured and calculated points (and the extrapolated points) in the radial and tangential directions are interpolated at each time step, to the face-centres at the draft tube inlet. It should be noted that the integrated inlet volume flow differs between the measured data and the computed data, and yet also for the different resolutions of the numerical inlet data. At this point it is shown that other things are much more important.

Figure 3 shows a circumferentially averaged representation of the different boundary conditions. It can be seen that the experimental and numerical data differ significantly in terms of the axial velocity component. They differ in terms of magnitude, but more importantly they differ in the region close to the hub, where the experimental data fails to include the effect of the hub clearance. It should be noted the difference between two coarse angular resolved numerical boundary conditions, where one fails to include some of the effect of the hub clearance. This difference is shown to give a significant difference in the draft tube flow.

\section{Codes and numerical settings}


The simulations have been done using two industrially used CFD codes, OpenFOAM-1.5-dev and ANSYS CFX 13 [7]. Both codes are based on the unstructured finite volume method, with a segregated solver in OpenFOAM and a coupled solver in CFX. A second-order backward time discretization scheme was used in both codes. The convection schemes for velocities was second-order linear upwind in OpenFOAM and High Resolution in CFX, while that of the turbulence quantities were first-order upwind in both codes. The time step was chosen so that the inlet boundary/runner would rotate one tangential inlet boundary face each time step, yielding approximate maximum CFL numbers of 5 and 45 in OpenFOAM and CFX, respectively.

\section{Results}

Figure 4 shows the results at sections $\mathrm{Ib}$ and Ic, from the simulations using the angular resolved experimental inlet data (Exp), the computed symmetric inlet data (Comp. symmetric), the computed angular resolved data with coarse resolution (Comp. resolved coarse), the computed angular resolved data with coarse resolution but with refined boundary layers (Comp. resolved coarse refined bl.) The numerical results from OpenFOAM and ANSYS CFX are compared with the experimental data. The experimental inlet data seems inadequate for use as inlet boundary condition. Both codes give poor results further down in the draft tube. They give quite different results in their attempts to repair the inlet condition, which seems poor. For the computed symmetric inlet data, both codes give similar results except in the region near the axis of rotation. Those differences are of such a level that the effects of turbulence model and numerical schemes etc. can be started to be evaluated. The results for the coarse computed angular resolved inlet data differ significantly from the symmetric case. The reason is attributed to the lack of clearance effects (c.f. figure 3). CFX seems to be more sensitive to this than OpenFOAM, in the present case. The results for the coarse computed angular resolved inlet data, where additional attention has been paid to the resolution of the clearance effects, come back to being similar to the symmetric case for both codes.

Figure 5 shows all the results from the OpenFOAM simulations using the numerical inlet data in the same plots. The computed results with the fine angular resolved inlet data are included, which could not be performed in CFX. The results from the different boundary conditions are similar except for the coarse angular resolved case. This highlights the importance of the clearance flow in the draft tube, and suggests that the unsteady effects of the runner blade wakes are not of equal importance for the featured studied here.

Figures 6 and 7 show the phase-averaged results at cross-sections Ib and Ic, for the case with coarse angular resolved inlet data and resolved boundary layers. The simulations preserve the effects of the runner blades to a reasonable level until cross-section $\mathrm{Ib}$. A finer mesh, aligned with the flow direction, is required in the region between cross-sections Ib and Ic to preserve the wakes further [8]. It should be recalled that the experimental and numerical data have different volume flow, making it difficult to do a quantitative comparison of the axial velocity distributions, and some differences in the contour plots when using the same colour legend. 

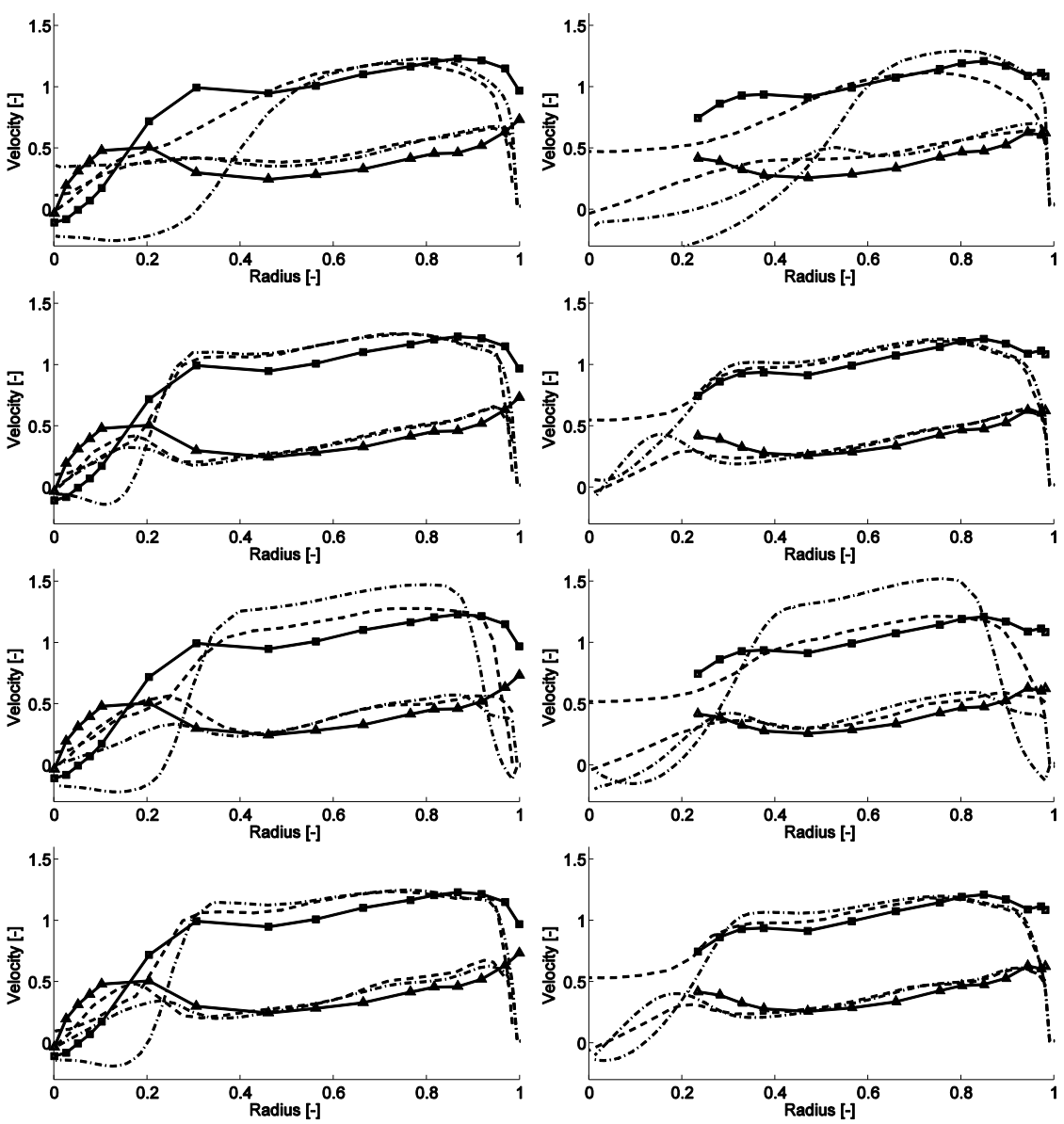

Figure 4. Normalized velocity distributions at sections Ib (left) and Ic (right), using inlet conditions from: Row 1: Exp., Row 2: Comp. symmetric, Row 3: Comp. resolved coarse, Row 4: Comp. resolved coarse refined bl. Solid lines: Experimental data (squares: axial, triangles: tangential). Dashed lines: OpenFOAM. Dash-dotted lines: CFX. 

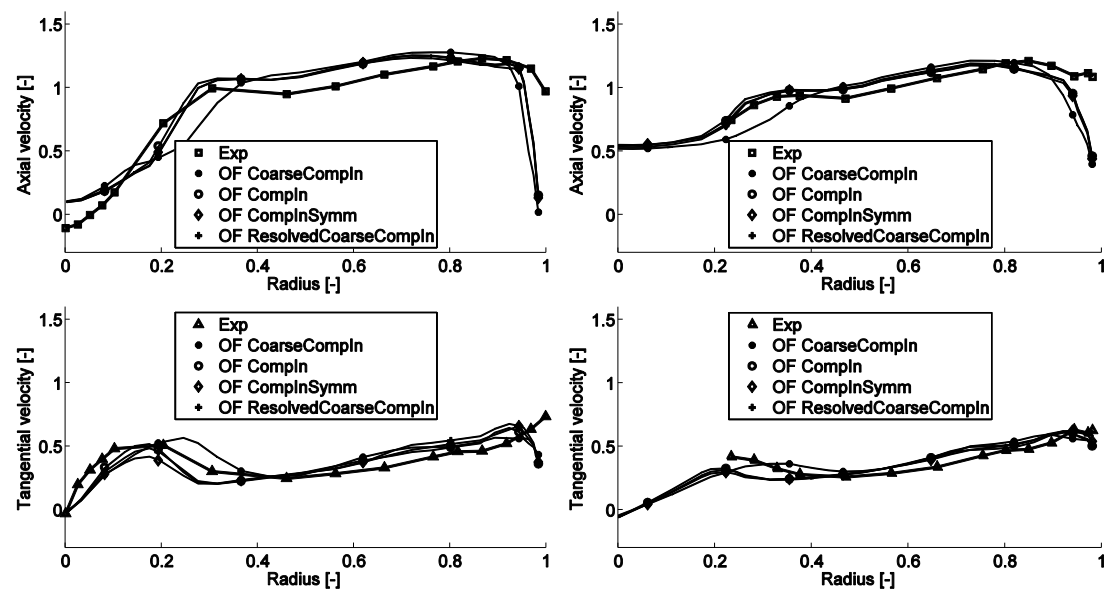

Figure 5. Normalized axial (top) and tangential (bottom) velocity distributions from OpenFOAM, at sections Ib (left) and Ic (right), using different inlet conditions.
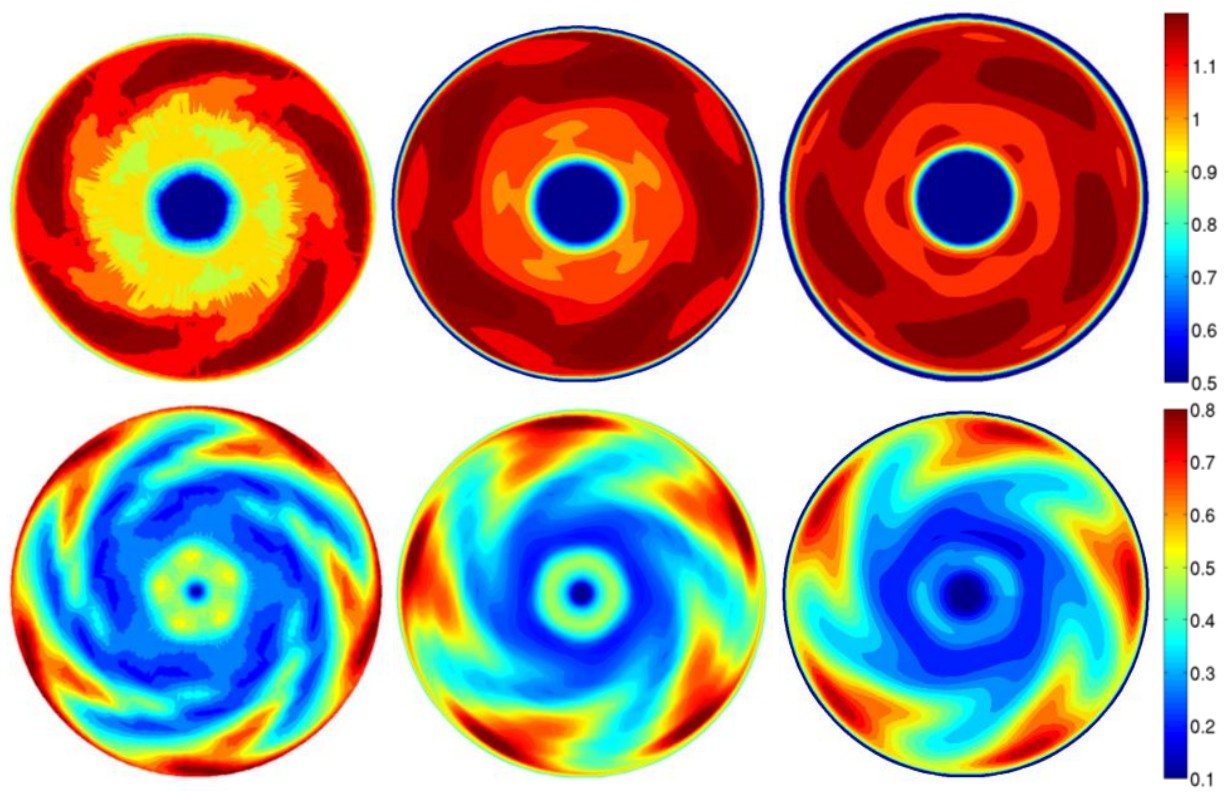

Figure 6. Measured and computed phase-averaged normalized velocity distribution at section Ib. Left: Measured, Center: OpenFOAM, Right: CFX. Top: Axial. Bottom: Tangential. 

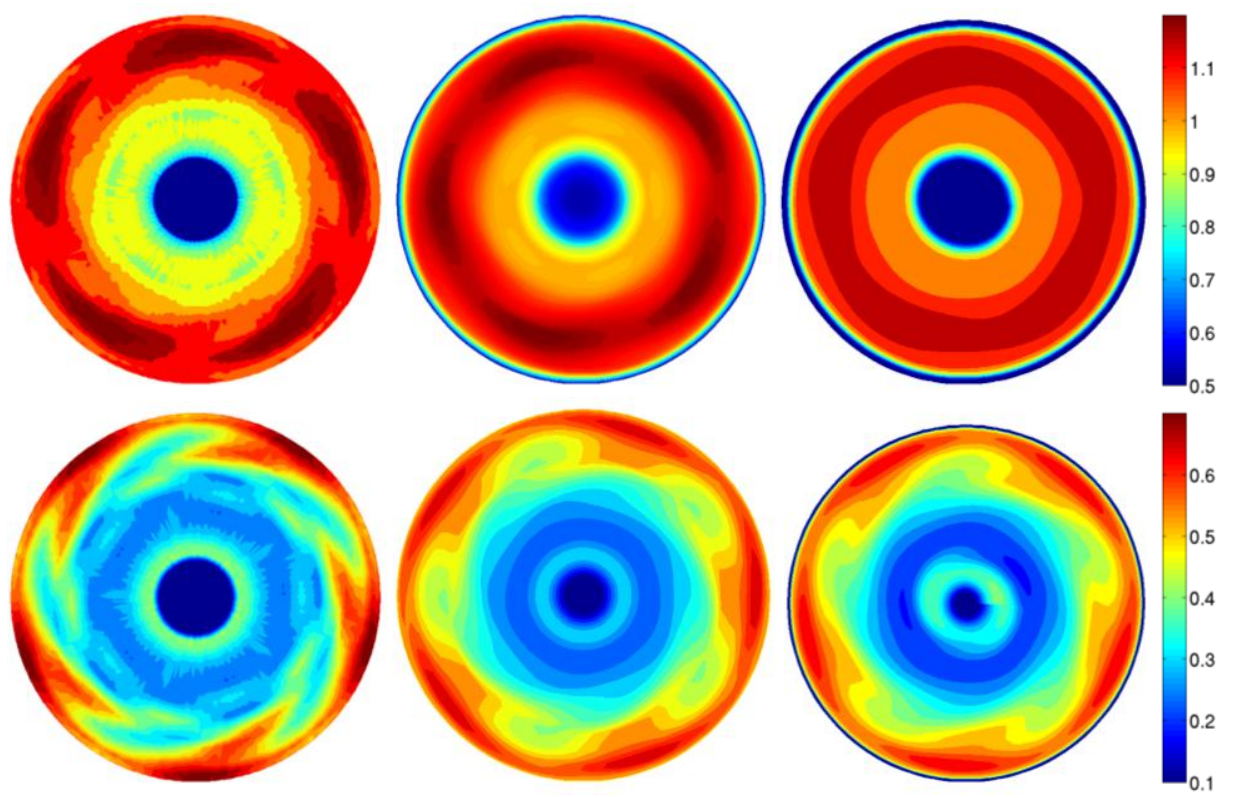

Figure 7. Measured and computed phase-averaged normalized velocity distribution at section Ic. Left: Measured, Center: OpenFOAM, Right: CFX.

Top: Axial. Bottom: Tangential.

\section{Conclusions}

The present results suggest that the experimental angular inlet boundary conditions proposed to the workshop are not adequate to simulate accurately the flow in the T-99 draft tube. Interpolated values from a runner simulation give better results. It is shown that the resolution of the local effects of the circumferentially averaged profiles, close to the hub and shroud (clearance flow), are much more important for the flow profiles at sections $\mathrm{Ib}$ and Ic than the inclusion of the effects of the runner blades. For the cases when the inlet boundary is properly resolved, OpenFOAM and CFX give very similar results except below the hub. CFX is much more sensitive to the resolution of the inlet data.

\section{Acknowledgements}

The research presented in this work was carried out as a part of the Swedish Hydropower Centre (SVC). SVC has been established by the Swedish Energy Agency, Elforsk and Svenska Kraftnät together with Luleå University of Technology, The Royal Institute of Technology, Chalmers University of Technology and Uppsala University. www.svc.nu.

\section{References}

[1] Cervantes M J, Engström F and Gustavsson H 2005 Proc. of the Turbine-99 III Workshop on Draft Tube Flow( Porjus, Sweden, 8-9 December 2005) Luleå University of Technology, 2005:20, ISSN:1402-1528

[2] Kurosawa S and Nakamura K 2005. Proc. of the Turbine-99 III Workshop on Draft Tube Flow, (Porjus, Sweden, 8-9 December2005) Luleå University of Technology, 2005:20, ISSN:14021528

[3] Cervantes M J, Andersson U and Lövgren M.. Turbine-99 unsteady simulations : validation 25th IAHR Sym. on Hydraulic machinery and systems(Timișoara, Romania)

[4] Andersson U and Cervantes M J 2008. Phase resolved velocity measurements at the draft tube cone of the turbine-99 test case. Hydro technology and the evironment for the new century: 24th IAHR Sym.on Hydraulic Machinery and Systems( Foz do Iguassu, Brazil)

[5] Nilsson H 2006 Evaluation of OpenFOAM for CFD of turbulent flow in water turbines. Proc. 
of 23rd IAHR Symposium(Yokohama, Japan)

[6] Bergström J 1999. Proc. of Turbine 99 - Workshop on Draft Tube Flow(Porjus, Sweden, June 20-23, 1999) Luleå University of Technology, 2000:11, ISSN:1402-1536

[7] ANSYS CFX 13.0, help manual

[8] Nilsson H 2002Numerical Investigations of Turbulent Flow in Water Turbines Ph.D Thesis (Sweden :Chalmers University of Technology) 